\title{
The effects of age and environment on the expression of inbreeding depression in Eucalyptus globulus
}

\author{
J Costa e Silva ${ }^{1,2}$, C Hardner ${ }^{3}$, P Tilyard ${ }^{4}$ and BM Potts ${ }^{4}$ \\ ${ }^{1}$ Centro de Estudos Florestais, Departamento de Ambiente, Território e Recursos Naturais, Instituto Superior de Agronomia, \\ Universidade Técnica de Lisboa, Lisboa, Portugal; ${ }^{2}$ Centro de Matemática e Aplicações, Departamento de Matemática, Instituto Superior \\ Técnico, Universidade Técnica de Lisboa, Lisboa, Portugal; ${ }^{3}$ School of Land, Crop and Food Sciences, University of Queensland, St Lucia, \\ Queensland, Australia and ${ }^{4}$ School of Plant Science and Cooperative Research Centre for Forestry, University of Tasmania, Hobart, \\ Tasmania, Australia
}

Inbreeding adversely affects fitness traits in many plant and animal species, and the magnitude, stability and genetic basis of inbreeding depression (ID) will have short- and longterm evolutionary consequences. The effects of four degrees of inbreeding (selfing, $f=50 \%$; full- and half-sib matings, $f=25$ and $12.5 \%$; and unrelated outcrosses, $f=0 \%$ ) on survival and growth of an island population of Eucalyptus globulus were studied at two sites for over 14 years. For selfs, ID in survival increased over time, reaching a maximum of $49 \%$ by age 14 years. However, their inbreeding depression for stem diameter remained relatively stable with age, and ranged from 28 to $36 \%$ across years and sites. ID for survival was markedly greater on the more productive site, possibly due to greater and earlier onset of inter-tree competition, but was similar on both sites for the diameter of survivors. The deleterious trait response to increasing inbreeding coefficients was linear for survival and diameter. Nonsignificant quadratic effects suggested that epistasis did not contribute considerably to the observed ID at the population level. Among- and within-family coefficients of variation for diameter increased with inbreeding degree, and the variance among the outcrossed families was significant only on the more productive site. The performance of self-families for diameter was highly stable between sites. This suggests that, for species with mixed mating systems, environmentally stable inbreeding effects in open-pollinated progenies may tend to mask the additive genotype-by-environment interaction for fitness traits and the adaptive response to the environment. Heredity (2011) 107, 50-60; doi:10.1038/hdy.2010.154; published online 12 January 2011

Keywords: outcrossing; selfing; inbreeding depression; additive and non-additive genetic effects; genotype-by-environment interaction; age trends

\section{Introduction}

Inbreeding adversely affects fitness traits in many plant and animal species (Husband and Schemske, 1996; Keller and Waller, 2002; Charlesworth and Willis, 2009). This phenomenon, termed inbreeding depression (ID), impacts on many evolutionary processes and is a key genetic factor causing the short-term loss of fitness in small populations (Charlesworth and Charlesworth, 1987; Lynch and Walsh, 1998, Chapter 10; Keller and Waller, 2002; Charlesworth and Willis, 2009). In plants, the magnitude of ID is related to the mating system, with predominantly outcrossing species exhibiting greater ID than those that predominantly self-fertilize (Husband and Schemske, 1996). The most commonly studied ID is due to self-fertilization, for which the expected inbreeding coefficient $(f)$ is 50\% (Husband and Schemske, 1996; Charlesworth and Willis, 2009). However, with often strong spatial structure in wild plant populations due to limited seed and/or pollen dispersal, milder inbreeding

Correspondence: Dr J Costa e Silva, Centro de Estudos Florestais, Departamento de Ambiente, Território e Recursos Naturais, Instituto Superior de Agronomia, Universidade Técnica de Lisboa, Tapada da Ajuda, Lisboa 1349-017, Portugal.

E-mail: jces@isa.utl.pt

Received 29 April 2010; revised 13 September 2010; accepted 15 November 2010; published online 12 January 2011 $(f<50 \%)$ may occur following crossing among related plants growing in close proximity (biparental inbreeding; see Hardner et al., 1998, for example).

ID is thought to be caused by two main non-mutually exclusive genetic mechanisms: directional dominance, where homozygotes produced by inbreeding unmask recessive and/or partially recessive deleterious alleles; and overdominance, where the heterozygote itself is advantageous (Charlesworth and Charlesworth, 1987; Lynch and Walsh, 1998, Chapter 10; Charlesworth and Willis, 2009). With the dominance mode, a population's genetic load may be more readily purged by natural selection compared with overdominance (Lande and Schemske, 1985; Charlesworth and Charlesworth, 1987). The effects and levels of dominance will affect the rate of purging, with recessive lethals more readily purged than partially recessive alleles with mild detrimental effects (Ritland, 1996; Charlesworth and Willis, 2009). A growing number of reports in plants support the dominance hypothesis (Charlesworth and Charlesworth, 1999; Carr and Dudash, 2003; Charlesworth and Willis, 2009), although some studies have suggested that overdominance and even epistasis constitute key mechanisms responsible for ID (Charlesworth and Willis, 2009). Epistasis contributes to ID only in the presence of directional dominance, and through interactions between loci involving additive $\times$ dominance and dominance $\times$ dominance effects (Crow and Kimura, 
1970, pp 81-83; Lynch and Walsh, 1998, Chapter 10). Quadratic regression analysis of fitness traits on expected inbreeding coefficients $(f)$ has been proposed to evaluate the overall trait depression (the linear effect) and the presence of epistasis (the curvilinear effect) (Crow and Kimura, 1970, pp 81-83). In this sense, a significant negative curvilinear term indicates that epistasis acts with a reinforcing effect, while a significant positive curvilinear term suggests a diminishing effect from epistasis and/or purging of deleterious recessive alleles (Crow and Kimura, 1970, pp 81-83).

As with any genetic effect, the magnitude of ID can also be affected by environmental factors, with fitness traits often exhibiting inbreeding-by-environment interaction (Lynch and Walsh, 1998, Chapter 10). Both abiotic (Brown et al., 2009) and biotic (Schmitt and Ehrhardt, 1990; Botham et al., 2009) factors can contribute to the differential expression of ID across environments. In a review of 34 studies, Armbruster and Reed (2005) found a trend for ID to increase in more stressful environments. However, this trend is not always evident (Armbruster and Reed, 2005; Waller et al., 2008).

In the present study, we compare the effects of multiple degrees of inbreeding on the survival and growth of Eucalyptus globulus Labill., an Australian forest tree, grown in two contrasting environments. We specifically aim to (1) compare the magnitude and pattern of the expression of ID in survival and growth across ages and environments; (2) assess whether epistasis contributes to ID for survival and growth at the population level; and (3) determine whether inbreeding effects on the among- and within-family components of variation for growth are stable across ages and environments.

\section{Materials and methods}

\section{Study species}

E. globulus (Tasmanian blue gum) is a forest tree native to south-eastern Australia (including Tasmania and King Island), but is widely grown in pulpwood plantations in temperate parts of the world. It is mainly pollinated by birds and insects, and has a mixed mating system with average outcrossing rates in undisturbed native forests $>85 \%$ (Mimura et al., 2009). Progeny derived from self-fertilization exhibit severe ID for growth and survival (Hardner and Potts, 1995; Hardner et al., 1998; Costa e Silva et al., 2010a), and there is also evidence for ID following biparental inbreeding in the wild (Hardner et al., 1998)

\section{Plant material and crossing design}

First-generation E. globulus full-sib families, derived from a factorial design involving base population parents from King Island trees, randomly selected in native stands (pollen parents) or a seed orchard (seed parents, chosen from different families remaining in a growth-culled progeny trial based on accessibility of flowers for crossing) (Volker et al., 2008), were used to generate second-generation full-sib families with four degrees of inbreeding: $f=0 \%$ from outcrosses, $f=12.5 \%$ from crosses among half sibs, $f=25 \%$ from matings among full sibs and $f=50 \%$ from selfing. The base population parents are assumed to be unrelated and non-inbred. Four sets of four full-sib families were selected from the first-generation factorial design. Two individuals were then randomly chosen from each of these families, so that four pollen parents and four seed parents from each set could be used for controlled crossing. These individuals were self-pollinated to produce selfed offspring (SELF, $f=50 \%$ ). Full-sib inbreeding (FSI, $f=25 \%$ ) was achieved by crossing seed and pollen parents within each of the first-generation full-sib families. Within each of the four sets, seed and pollen parents that shared one common base population parent were crossed to obtain half-sib inbred progeny (HSI, $f=12.5 \%$ ). Pollen and seed parents within each set that had no common base population parents were crossed to provide second-generation outcrossed families (OUT, $f=0 \%$ ).

The four sets of first-generation full-sib families comprised a total number of 11 base population parents (eight used as pollen parents and three used as seed parents). As each of the two individuals chosen within a full-sib family was employed only once as a parent for a given second-generation cross type, this would lead to 16 families for OUT, HSI and FSI, and 32 families for SELF. However, due to crossing failure, only 54 crosses were successful, leading to 10, 10, 11 and 23 families (corresponding to percentages of non-failures of 63, 63, 69 and $72 \%$ ) for the OUT, HSI, FSI and SELF cross types, respectively. Thus, the distribution of base population parents to the various cross types was unbalanced, but all the 11 founder trees contributed progeny to each cross type.

\section{Field experiments}

Progenies from the successful crosses were planted in two field trials, located at west Ridgley (latitude $41^{\circ} 10^{\prime} 28^{\prime \prime}$, longitude $145^{\circ} 46^{\prime} 22^{\prime \prime}$ ) and Southport (latitude $43^{\circ} 22^{\prime} 14^{\prime \prime}$, longitude $146^{\circ} 56^{\prime} 31^{\prime \prime}$ ), in northern and southern Tasmania, respectively. Ridgley was an ex-pasture site with a high-quality clay loam kraznozem soil, having little variability over the trial area. Southport was a wet ex-forest site with a poor sandy clay soil, exhibiting reduced drainage in some areas. Both of the plantation sites had a slight-to-moderate slope, and were located at an altitude of $\sim 280$ and $130 \mathrm{~m}$ in Ridgley and Southport, respectively. The site preparation included rip mounding in both trials, and pre-planting herbicide treatments in Ridgley. Tree spacing was $2.5 \times 3.5 \mathrm{~m}$ and $2.5 \times 4 \mathrm{~m}$ in Ridgley and Southport, respectively.

The experimental layout in both trials was a randomized block design. Seedlings from the families of a cross type were planted as single-tree non-contiguous plots, randomized within each of the 20 replicates per trial. This was intended to reproduce the mixture of offspring of the various cross types, which may occur under natural conditions in native stands. All the families from a cross type were represented in both trials, except for one family from full-sib crossing that was planted at Southport only.

Survival (dead/alive) was recorded for each individual tree, and the proportion of planted trees alive per replicate (SURV) was calculated for each cross type. Over-bark diameter at breast height (DBH) was measured on surviving trees. Measurement ages from planting were 2, 4, 6, 10 and 14 years for Ridgley, and 2, 4, 6, 8 and 14 years for Southport. SURV and DBH are both fitness components, although total fitness is 
expected to be more closely correlated to survival than to growth rate (Crnokrak and Roff, 1999; Armbruster and Reed, 2005).

\section{Data analysis}

Data analyses were undertaken for each trial and age following the general linear mixed model:

$$
\mathbf{y}=\mathbf{X b}+\mathbf{Z}_{\mathbf{0}} \mathbf{u}_{\mathbf{0}}+\mathbf{Z}_{\mathbf{g}} \mathbf{u}_{\mathbf{g}}+\mathbf{e}
$$

where $\mathbf{y}$ is a $N \times 1$ combined vector of observations over cross types for a given trait $\left(N=\sum_{i=1}^{p} N_{i}\right.$, where $N_{i}$ is the number of observations in the $i$ th cross type, $i=1, \ldots, p$ and $p=4$ cross types), $\mathbf{b}$ is a $l \times 1$ vector of fixed effects with a $N \times l$ incidence matrix $\mathbf{X}, \mathbf{u}_{\mathbf{g}}$ is a $n_{i} p \times 1$ vector for the random effects of $n_{i}$ families within each of $p$ cross types with a $N \times n_{i} p$ incidence matrix $\mathbf{Z}_{\mathbf{g}}, \mathbf{u}_{\mathbf{0}}$ is a $m \times 1$ vector involving additional random effects (such as experimental design features) with a $N \times m$ incidence matrix $\mathbf{Z}_{0}$, and $\mathbf{e}$ is a $N \times 1$ vector of residual terms for each of $p$ cross types. The data vector $(\mathbf{y})$ contained observations at the replicate level for SURV or at the individual tree level for DBH. Only survival recorded after age 4 years was used in the analysis of SURV, as the overall incidence of mortality was low for all cross types at ages 2 and 4 years.

The analyses of SURV and DBH incorporated inbreeding effects into the mixed model either as: (1) a class variable, with the terms in $\mathbf{b}$ comprising the overall trial mean and the effects of cross type, to test for pair-wise differences between the means of the cross types; or as (2) a continuous variable, so that the relationship between trait performance and $f$ is modelled to test whether ID is linearly related to the increase in inbreeding coefficient. Under the latter parameterization, besides an intercept, the terms in $\mathbf{b}$ involved regression coefficients that included either linear or quadratic polynomial functions of the $f$ values in $\mathbf{X}$. The family effects in $\mathbf{u}_{\mathbf{g}}$ were fitted under the analysis of DBH only. The vector $\mathbf{u}_{\mathbf{0}}$ comprised replicate effects in the analyses of both traits, and effects due to the interaction between replicates and cross types for DBH.

Under the linear mixed model defined in expression (1), the joint distribution of the random terms was assumed to be multivariate normal, with the mean vector and variance matrix defined as

$$
\left[\begin{array}{l}
\mathbf{u}_{\mathbf{0}} \\
\mathbf{u}_{\mathbf{g}} \\
\mathbf{e}
\end{array}\right] \sim N\left(\left[\begin{array}{l}
\mathbf{0} \\
\mathbf{0} \\
\mathbf{0}
\end{array}\right],\left[\begin{array}{ccc}
\mathbf{G}_{\mathbf{0}} & \mathbf{0} & \mathbf{0} \\
\mathbf{0} & \mathbf{G}_{\mathbf{g}} & \mathbf{0} \\
\mathbf{0} & \mathbf{0} & \mathbf{R}
\end{array}\right]\right)
$$

where $\mathbf{G}_{\mathbf{0}}, \mathbf{G}_{\mathbf{g}}$ and $\mathbf{R}$ are variance matrices concerning $\mathbf{u}_{\mathbf{0}}$, $\mathbf{u}_{\mathbf{g}}$ and $\mathbf{e}$, respectively. In the analysis of $\mathrm{DBH}$, separate model terms and family effects among cross types were assumed to be independent in $\mathbf{G}_{\mathbf{0}}$ and $\mathbf{G}_{\mathbf{g}}$, respectively, and thus $\mathbf{G}_{\mathbf{0}}=\oplus_{j=1}^{q} \mathbf{G}_{\mathbf{0}_{j}}=\oplus_{j=1}^{q} \sigma_{j}^{2} \mathbf{I}_{n_{j}}$ and $\mathbf{G}_{\mathbf{g}}=\oplus_{i=1}^{p} \sigma_{f_{i}}^{2} \mathbf{I}_{n_{i}}$, where $\mathbf{G}_{0}$ is the variance matrix for the $j$ th random term $(j=1, \ldots, q$, and $q=2$ pertaining to replicates and the interaction between replicates and cross types), $\sigma_{j}^{2}$ is the variance associated with the $j$ th model term, $\sigma_{f}^{2}$ is the variance among families for the ith cross type, $\mathbf{I}_{n_{i}}$ and $\mathbf{I}_{n}$ are identity matrices with dimensions $n_{i} \times n_{i}$ $\left(n_{i}=\right.$ number of families in the $i$ th cross type) and $n_{j} \times n_{j}$ ( $n_{j}=$ number of levels in the $j$ th term), and $\oplus$ is the direct sum operation. For both traits, the residual effects from different cross types were modelled as being independent, and thus $\mathbf{R}$ was defined as $\oplus_{i=1}^{p} \sigma_{e_{i}}^{2} \mathbf{I}_{N_{i}}$ where, for the $i$ th cross type, $\sigma_{e}^{2}$ is the residual variance (for $\mathrm{DBH}, \sigma_{e}^{2}$ corresponds to the within-family variance) and $\mathbf{I}_{N_{i}}$ is a identity matrix of dimension $N_{i} \times N_{i}$. Therefore, heterogeneity of variances among cross types for effects in $\mathbf{u}_{\mathbf{g}}$ and $\mathbf{e}$ was accounted for in the data analysis.

The variance components were estimated by restricted maximum likelihood, using the average information algorithm developed by Gilmour et al. (2006). For DBH, the significance of the estimated $\sigma_{f}^{2}$ was assessed using a one-tailed likelihood ratio (LR) test (Stram and Lee, 1994). Initial analyses of DBH indicated that the variance estimate associated with the interaction between replicates and cross types was small and not statistically different from zero $(P>0.05$, according to a LR test) at all measurement ages in both trials, and thus this random term was dropped from the model in subsequent analyses. The estimation of a family correlation between sites for $\mathrm{DBH}$ within a cross type was attempted under an analysis combining data from both trials, and using an unstructured variance-covariance matrix (with among-family variances within trials on the diagonal and a family covariance between trials on the offdiagonal) for a given cross type in $\mathbf{G}_{\mathbf{g}}$. This extension of the mixed model to include a covariance matrix between trials for a given cross type was pursued only for cross types where a statistically significant $(P \leqslant 0.05)$ family variance has been detected in both trials.

For each trait at a given age and trial, least-squares means (LSM) and standard errors of differences between LSM were estimated for each cross type and pairs of cross types, respectively. Pair-wise differences between LSM were then tested using a $t$-test, and by applying the Kenward and Roger (1997) approximation for calculating the degrees of freedom associated with the test statistic. Population level ID due to several degrees of inbreeding was expressed on a percentage basis as:

$$
\mathrm{ID}_{i}=\left(\mathrm{LSM}_{(\mathrm{OUT})}-\mathrm{LSM}_{(i)}\right) / \mathrm{LSM}_{(\mathrm{OUT})} \times 100
$$

where $\operatorname{LSM}_{(\mathrm{OUT})}$ and $\mathrm{LSM}_{(i)}$ are the least-squares means for the OUT and $i$ th cross types, respectively, with $i$ being HSI, FSI or SELF; the pair-wise differences between the LSM of OUT versus that of HSI, FSI or SELF provide significance tests of non-zero values for ID expressed on an absolute scale (that is, $\operatorname{LSM}_{(\mathrm{OUT})}-\mathrm{LSM}_{(i)}$ ).

For the regression of trait performance on the expected inbreeding coefficients at each age and trial, the linear and quadratic terms were fitted sequentially in the model, and their significance was tested by incremental Wald-type F-statistics using the Kenward and Roger (1997) adjustments to compute approximate denominator degrees of freedom. A significant negative linear regression is consistent with both the dominance and overdominance hypotheses for the genetic basis of ID (Lynch and Walsh, 1998, Chapter 10). In this sense, the estimated linear regression coefficient $(\hat{\beta})$ provides a measure of the trait response to reduced heterozygosity from a unit ( $1 \%$ point) increase in $f$. A departure from linearity (as detected by a significant quadratic regression) suggests that directional epistasis involving dominance effects may also contribute to ID at the population level (Crow and Kimura, 1970, pp 81-83; Lynch and Walsh, 1998, Chapter 10). As for the ID values, the linear $\hat{\beta}$ coefficients were adjusted to compare parameter estimates across traits, ages and trials. This was done by dividing $\hat{\beta}$ by the LSM $_{(\mathrm{OUT})}$ of a trait at a certain age 
and trial. With the adjusted $\hat{\beta}\left(\hat{\beta}_{\text {adj }}\right)$, the trait response per $1 \%$ point increase in $f$ is given in percentage of the outcrossing population mean.

An analysis combining data across trials was conducted for each trait to test whether differences between trial means were significant at a comparable age for a given cross type. The mixed model applied was an extension of that used for single trials. Effects in $\mathbf{b}$ included the overall mean, trial site, cross type, and the interaction between trial site and cross type. The random terms were assumed to be independent across trials, and the mixed model incorporated heterogeneity of variances among trials and cross types for effects in $\mathbf{u}_{\mathrm{g}}$ in the analysis of $\mathrm{DBH}$, and for effects in $\mathbf{u}_{\mathbf{0}}$ and $\mathbf{e}$ in the analyses of both traits. For a given trait, LSM were estimated for each cross type and trial, and pair-wise differences between trials in LSM were tested for each cross type by following a procedure similar to that described above for single sites. Subsequently, another combined analysis was performed to test whether a trait response to increasing inbreeding coefficients differed between the two trials at comparable ages. The effects in $\mathbf{u}_{0}, \mathbf{u}_{\mathrm{g}}$ and $\mathbf{e}$ were fitted as defined for the previous combined data analysis. Based on results from the singletrial analyses (see below), the model using the combined information included only linear regression effects in $\mathbf{b}$. In this sense, the fixed terms in $\mathbf{b}$ fitted the model $\alpha_{2}+\left(\alpha_{1}-\alpha_{2}\right)+\beta_{2} f+\left(\beta_{1}-\beta_{2}\right) f$, where $\alpha$ and $\beta$ denote the intercept and slope, and the subscripts 1 and 2 refer to the Ridgley and Southport trials, respectively; $f$ is the regressor variable, defined as before. Under this parameterization of the fixed part of the mixed model, the heterogeneity of slopes between trials is tested through the deviation $\beta_{1}-\beta_{2}$. This was carried out using a Wald F-test (with incremental and conditional Wald-type F-statistics giving the same results for testing $\beta_{1}-\beta_{2}$ ), with the Kenward and Roger (1997) adjustments in the denominator degrees of freedom. A significant heterogeneity of slopes will lead to the rejection of the null hypothesis that the trait response to inbreeding is independent of the trial site. Before testing for the heterogeneity of slopes between trials, the DBH data were centered and standardized. The applied scale transformation involved taking the difference between each record and the trial mean, and then expressing the differences in units of the phenotypic standard deviation of the individual observations in that trial. The difference between trials in DBH response to inbreeding was then tested using standardized regression coefficients, so that scaling effects arising from differences in site productivity could be accounted for when evaluating the significance of the heterogeneity of slopes.

Normality of the residuals within each cross type was evaluated with the Shapiro and Wilk (1965) test and the examination of quantile-quantile plots. Deviations from normality were particularly detected for SURV, although they were in general marginally significant as indicated by the Shapiro-Wilk test (in Ridgley, OUT at ages 6 and 10 years significant at the 1 and $5 \%$ levels, respectively; in Southport, HSI at age 6 years and OUT at age 8 years significant at the 5\% level in both cases). An arcsine transformation $\left(\sin ^{-1}(\sqrt{\mathrm{SURV}})\right)$ with a correction for a proportion of survival equal to one (Bartlett, 1947) improved the normality but, compared with the analysis using the untransformed SURV data, led to analogous results in terms of significance tests for pair-wise differences between cross-type LSM and regression coefficient estimates. Therefore, only the analysis based on untransformed values is presented for SURV.

Statistical analyses were carried out using ASReml (VSN International Ltd, Hemel Hempstead, UK) (Gilmour et al., 2006) and SAS (SAS Institute Inc., Cary, NC, 2004).

\section{Results}

\section{Comparisons of cross-type means}

In general, Southport tended to have higher SURV than Ridgley at the same age (Table 1). However, analyses combining SURV data across trials revealed significant differences between trial means only for the full-sib crosses (FSI, $P \leqslant 0.05$ and $P \leqslant 0.01$ at ages 6 and 14 years) and selfs (SELF, $P \leqslant 0.01$ and $P \leqslant 0.001$ at ages 6 and 14 years). The comparisons of cross-type LSM indicated a significant effect of inbreeding on SURV at age 6 years in both trials (Table 1). However, the development of significant differences in mortality between cross types was more rapid in Ridgley, as was the increase in mortality of the inbred populations with age. The highest rate of SURV decrease per year occurred in the selfs, with the decline being more pronounced in Ridgley. By age 14 years, all four cross types could be significantly discriminated in Ridgley, whereas in Southport only three groups of cross types (outcross, mild and high degrees of inbreeding) could be significantly differentiated.

Surviving trees in Ridgley generally had higher mean DBH than those in Southport (Table 2), and analyses combining DBH data from both trials showed that, at the same age, these differences were always highly significant $(P \leqslant 0.001)$ for a given cross type. A decline in DBH as a result of inbreeding was already evident at age 2 years (Table 2). From age 4 years onwards, the pattern of differences among cross types in the DBH of the surviving trees was consistent within a trial, but differed between trials. Indeed, from age 4 to 14 years, the discrimination among populations for the effects of inbreeding on the DBH of surviving trees was reverse to that observed for SURV, with a greater discrimination for DBH in Southport than in Ridgley. Thus, where the reduction of SURV due to inbreeding was more severe (that is, Ridgley), the effects of inbreeding on the DBH of surviving trees were reduced.

\section{Inbreeding depression due to different degrees of inbreeding}

ID for SURV increased from age 6 to 14 years within both trials (Table 1). Although the increase of ID with the degree of inbreeding tended to be consistent across ages within trials for SURV, this was more marked in Ridgley than in Southport. In addition, at comparable ages, Ridgley had stronger reductions in SURV than those observed in Southport for the FSI and SELF cross types.

ID in the DBH of surviving trees increased consistently with inbreeding degree but, in contrast to SURV (Table 1), the magnitudes did not change markedly with age (Table 2). For a given degree of inbreeding and age, ID in DBH differed only slightly between trials (for example, maximum differences of $4.9,2.2$ and $6.5 \%$ for $\mathrm{ID}_{\mathrm{HSI}}, \mathrm{ID}_{\mathrm{FSI}}$ and $\mathrm{ID}_{\mathrm{SELF}}$, respectively). Although ID in 
Table 1 Estimated least-squares means (LSM) and population-level inbreeding depression (ID), and results from the linear regression on expected inbreeding coefficients $(f)$ for percentage survival, based on progeny data from four cross types-outcrosses (OUT), half-sib inbreeding (HSI), full-sib inbreeding (FSI) and selfing (SELF)—in two E. globulus field trials (Ridgley and Southport) at different ages (in years) from planting

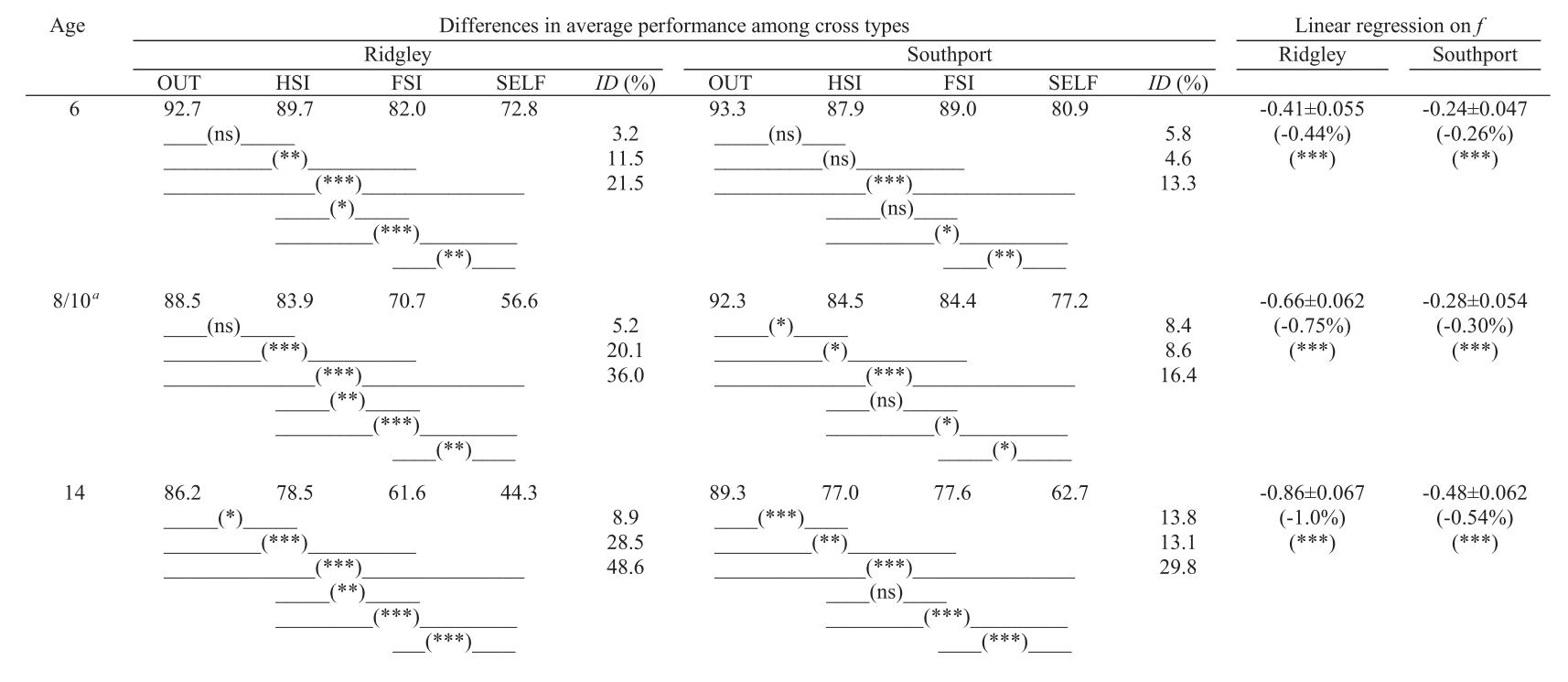

${ }^{\text {a Age }} 10$ years in Ridgley and 8 years in Southport.

The significance probabilities pertaining to the tests of differences between pairs of LSM are given for each trial and age. The ID values due to different degrees of inbreeding are aligned with the comparisons of pairs of LSM that were used in their calculation for each trial and age. The estimates of linear regression coefficients $(\beta)$ and their standard errors refer to the decline in percentage survival (with the reduction in survival relative to the LSM of the OUT cross type given in percentage within parentheses, and referred as $\beta_{\text {adj }}$ in the text) per $1 \%$ point increase in $f$. Significance probabilities from testing the null hypothesis $\beta=0$ are also shown within parentheses.

Non-significant (ns), $P>0.05$.

$* P \leqslant 0.05$.

${ }^{* *} P \leqslant 0.01$

$* * * P \leqslant 0.001$.

DBH following selfing ( (D $_{\text {SELF }}$ ) was always slightly higher in Southport than Ridgley, this was not the case for the milder degrees of inbreeding.

At age 6 years, inbreeding affected DBH more strongly than SURV in both trials. This difference generally remained in Southport, but at ages 10 and 14 years in Ridgley SURV showed higher levels of $\mathrm{ID}_{\mathrm{FSI}}$ and/or $\mathrm{ID}_{\text {SELF }}$ than DBH. However, for the mildest degree of inbreeding examined $\left(\mathrm{ID}_{\mathrm{HSI}}\right), \mathrm{DBH}$ was depressed more than SURV at these older ages.

\section{Relationship between trait performance and inbreeding coefficient}

The linear term of the quadratic regression analysis of trait performance on $f$ was highly significant $(P \leqslant 0.001$; Tables 1 and 2) and exhibited a negative relationship (Figures 1 and 2) for both SURV and DBH in the two trials at all ages, while the quadratic coefficient was not significant $(P>0.05)$. For SURV, the estimated linear regression coefficient $(\hat{\beta})$ and the adjusted coefficient value $\left(\hat{\beta}_{\text {adj }}\right)$ followed a similar pattern, becoming more negative with time within each trial (Table 1), consistent with ID for SURV increasing with age. Within each trial, the $\hat{\beta}$ values for $\mathrm{DBH}$ became more negative with age, but these changes parallel age-related differences in the overall means, and thus are likely to reflect scaling effects. Indeed, the changes in $\hat{\beta}_{\text {adj }}$ with age were not marked (ranging from -0.52 to $-0.58 \%$ in Ridgley, and from -0.61 to $-0.73 \%$ in Southport, per $1 \%$ point increase in $f$ ) for DBH (Table 2). Based on the $\hat{\beta}_{\text {adj }}$ coefficients, the expected ID for SURV at $f=50 \%$, depending upon age, ranged from 22 to $50 \%$ in Ridgley and from 13 to $27 \%$ in Southport; for $\mathrm{DBH}$, the corresponding ranges were $26-29 \%$ and $30-36 \%$, respectively. These regression-based estimates for ID are close

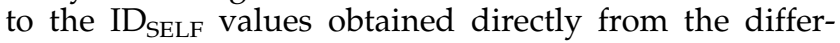
ences between cross-type LSM at the same age.

As departures from linearity were found to be not significant in each trial, testing the across-site heterogeneity of the trait responses to inbreeding was carried out by omitting a quadratic term from the mixed model using combined data across trials. For SURV, the difference between trials in $\hat{\beta}$ (Figure 1) was significant at the 5 and $0.1 \%$ levels at ages 6 and 14 years, respectively. This supports the ID results in that the reduction of SURV with inbreeding was more severe in Ridgley compared with Southport at the same age. As indicated by $\hat{\beta}$, the decline in $\mathrm{DBH}$ per $1 \%$ point increase in $f$ tended to be slightly more pronounced in Ridgley compared with Southport at the same age (Figure 2), but this pattern contrasted with that from $\hat{\beta}_{\text {adj }}$, which showed somewhat less ID in Ridgley (Table 2). For DBH, changes in the magnitude of raw $\hat{\beta}$ coefficients across trials are likely to have been influenced by scaling effects related to differences between trials in site productivity 
Table 2 Estimated least-squares means (LSM) and population-level inbreeding depression (ID), and results from the linear regression on expected inbreeding coefficients $(f)$ for diameter (DBH, in $\mathrm{cm}$ ), based on progeny data from four cross types-outcrosses (OUT), half-sib inbreeding (HSI), full-sib inbreeding (FSI) and selfing (SELF)—in two E. globulus field trials (Ridgley and Southport) at different ages (in years) from planting

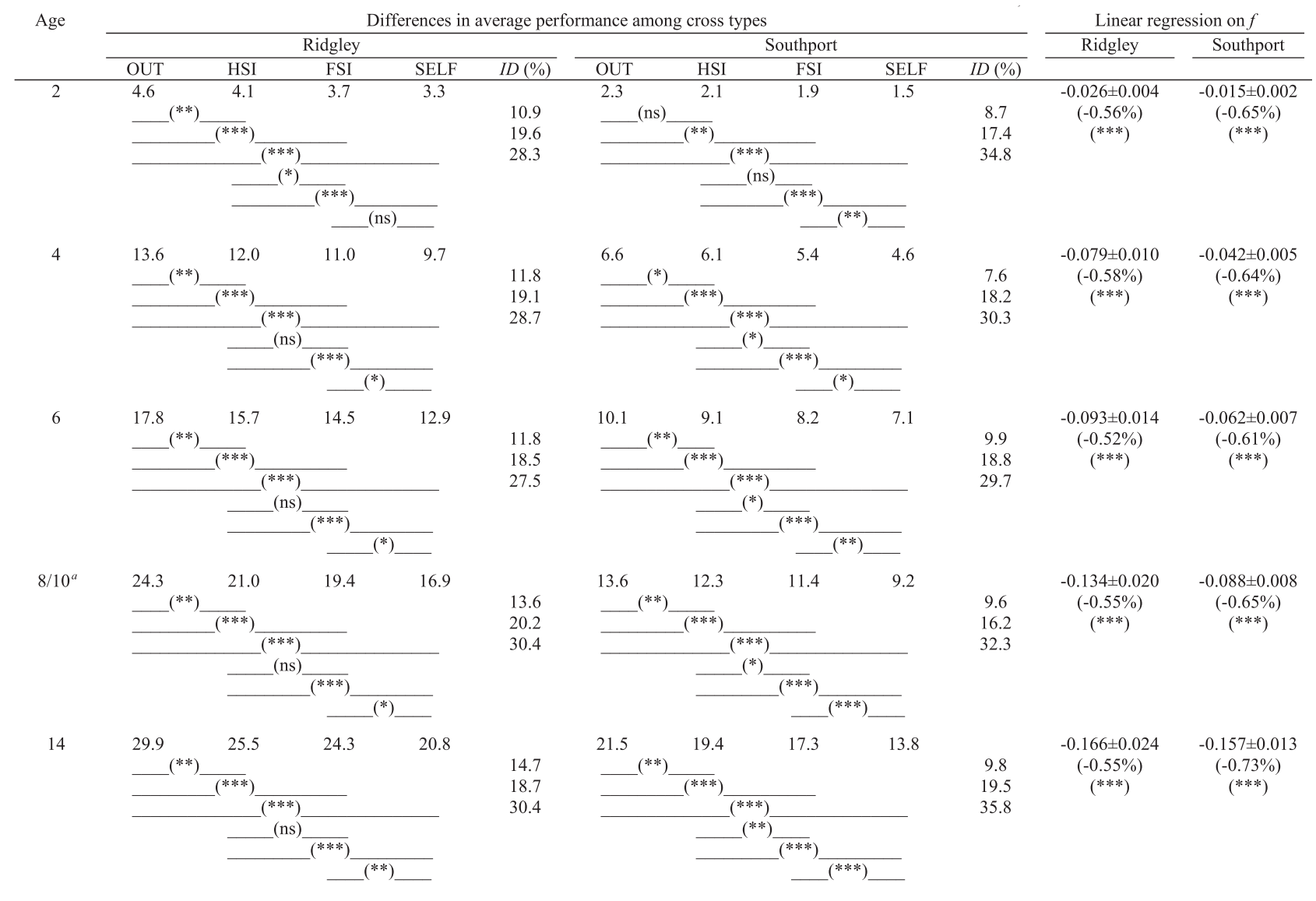

\footnotetext{
${ }^{\mathrm{a}}$ Age 10 years in Ridgley and 8 years in Southport.
}

The significance probabilities pertaining to the tests of differences between pairs of LSM are given for each trial and age. The ID values due to different degrees of inbreeding are aligned with the comparisons of pairs of LSM that were used in their calculation for each trial and age. The estimates of linear regression coefficients $(\beta)$ and their standard errors refer to the decline in DBH (with the reduction in DBH relative to the LSM of the OUT cross type given in percentage within parentheses, and referred as $\beta_{\text {adj }}$ in the text) per $1 \%$ point increase in $f$. Significance probabilities from testing the null hypothesis $\beta=0$ are also shown within parentheses.

Non-significant (ns), $P>0.05$.

$* P \leqslant 0.05$.

${ }^{* *} P \leqslant 0.01$.

${ }^{* * *} P \leqslant 0.001$.

(as indicated by the significantly higher cross-type LSM estimates in Ridgley compared with Southport at the same age). However, differences between trials in standardized $\hat{\beta}$ coefficients (which are scale invariant) were never significant $(P>0.05)$ for DBH. This suggests that the amount of ID in the DBH of survivors was comparable across sites, consistent with the small differences between trials in ID at comparable ages.

\section{$\mathrm{DBH}$ variation at various degrees of inbreeding}

Highly significant $(P \leqslant 0.001)$ variances among selfed families $\left(\hat{\sigma}_{f_{\text {SELF }}}^{2}\right)$ were usually detected in both trials (Table 3). This tendency was also observed in Ridgley for the other cross types $\left(\hat{\sigma}_{f_{\mathrm{OUT}}}^{2}\right.$ and $\hat{\sigma}_{f_{\mathrm{HSI}}}^{2}$, less so for $\left.\hat{\sigma}_{\mathrm{f}_{\mathrm{FSI}}}^{2}\right)$ but not in Southport, where the variation among families within outcrosses and mild degrees of inbreeding was generally found to be non-significant.

In general, the among-family $\left(\mathrm{CV}_{\mathrm{f}}\right)$ and within-family $\left(\mathrm{CV}_{\mathrm{wf}}\right)$ coefficients of variation increased with the degree of inbreeding in the two trials (Table 3). In Ridgley, the changes of $\mathrm{CV}_{\mathrm{f}}$ with age tended to differ among cross types: for OUT and HSI, $\mathrm{CV}_{\mathrm{f}}$ appeared to increase until age 10 years and then stabilized, whereas there was a tendency for $\mathrm{CV}_{\mathrm{f}}$ to decrease continuously with age for SELF; for FSI, $\mathrm{CV}_{\mathrm{f}}$ seemed to decrease until age 6 years and then stabilized. Thus, the difference between OUT and SELF in among-family variation was reduced with age. For within-family effects, the $\mathrm{CV}_{\mathrm{wf}}$ had a consistent pattern of a decrease followed by an increase with age for all cross types, although the increase occurred earlier for OUT and HSI (between ages 4 and 6 years) compared 

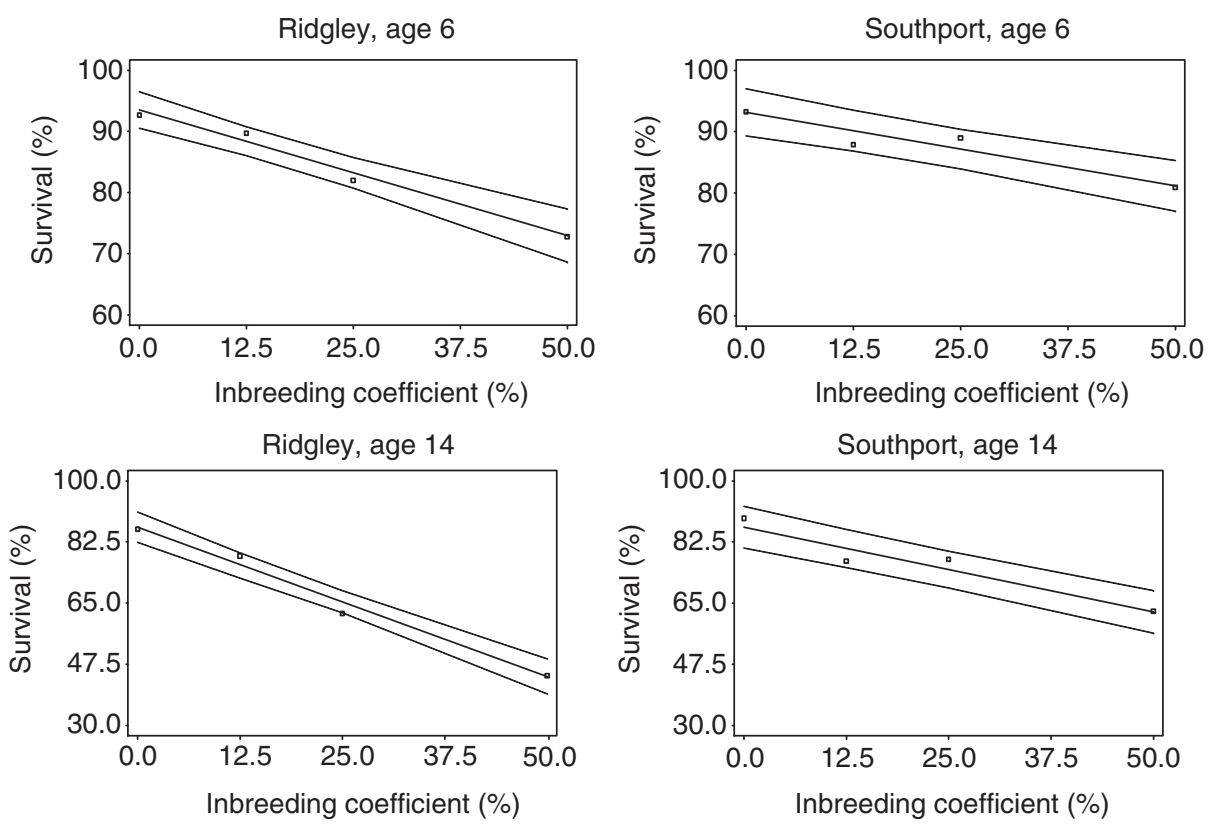

Figure 1 Fitted linear regressions (with the upper and lower bounds of $95 \%$ confidence intervals) of percentage survival on the expected inbreeding coefficients $(f)$ for the Ridgley and Southport trials at ages 6 and 14 years from planting. The squared symbols refer to least-squares means estimated for each of four cross types (see Table 1) corresponding to $f=0 \%$ (outcrosses), $12.5 \%$ (crosses among half sibs), $25 \%$ (matings among full sibs) and 50\% (selfing).

with FSI and SELF (between ages 6 and 10 years). The difference between OUT and SELF in $\mathrm{CV}_{\mathrm{wf}}$ was also reduced with age, and it seemed to stabilize from age 6 years onwards.

$\mathrm{CV}_{\mathrm{f}}$ also decreased with age for SELF in Southport, but appeared to stabilize earlier (from age 6 years onwards) than in Ridgley and was slightly higher at most ages (Table 3). A key difference between trials, however, was the fact that the $\mathrm{CV}_{\mathrm{f}}$ for the OUT progenies was virtually zero at all ages in Southport, suggesting that additive and non-additive genetic effects under random mating were not expressed in this trial. For the mild degrees of inbreeding, the $\mathrm{CV}_{\mathrm{f}}$ tended also to be lower in Southport compared with Ridgley at the same age, except for the HSI progenies at age 2 years. Another important difference between trials was the fact that Southport had higher $C V_{w f}$ than Ridgley for all cross types, suggesting a greater environmental heterogeneity within the ex-forest trial site. As indicated by the age trend in $\mathrm{CV}_{\mathrm{wf}}$, this environmental heterogeneity may have declined with age as plants became established in the field and, by age 14 years, there was little difference between trials in $\mathrm{CV}_{\mathrm{wf}}$ for all cross types. As in Ridgley, the difference between OUT and SELF in $C_{\text {wf }}$ was reduced with age in Southport, and appeared to stabilize from age 6 years onwards. Nevertheless, across all cross types, $\mathrm{CV}_{\mathrm{wf}}$ tended to decrease continuously with age in Southport (although with small changes after age 4 years), and thus the change with age differed from that observed in Ridgley.

Across-site family correlations of $0.99 \pm 0.09,0.98 \pm$ $0.06,0.83 \pm 0.13$ and $0.76 \pm 0.23$ at ages $2,4,6$ and 14 years were estimated for SELF families (data not shown). These high correlations indicate that the performance of SELF families was reasonably stable across trial sites, although the age trend of the estimates suggested that the magnitude of genotype-by-environment $(G \times E)$ interaction tended to increase with time. An extension of the mixed model to include a covariance matrix across trials for family effects in $\mathbf{G}_{\mathbf{g}}$ was not attempted for the other cross types because $\hat{\sigma}_{f_{\mathrm{OUT}}}^{2}, \hat{\sigma}_{f_{\mathrm{HSI}}}^{2}$ and $\hat{\sigma}_{\mathrm{frI}_{\mathrm{FI}}}^{2}$ were generally found to be non-significant in Southport (Table 3), and thus estimated across-site correlations would not be meaningful. Nevertheless, it is likely that $\mathrm{G} \times \mathrm{E}$ interaction is responsible for the large differences observed across sites in $\hat{\sigma}_{f_{\text {OUT }}^{2}}^{2}$ and, to a less extent, $\hat{\sigma}_{f_{\mathrm{HSI}}^{2}}^{2}$ and $\hat{\sigma}_{f_{\mathrm{FSI}}}^{2}$ (Table 3).

\section{Discussion}

The present study emphasizes the dynamic nature of ID with respect to the trait, age and environment. There was clearly an interplay between the two fitness traits SURV and $\mathrm{DBH}$, with size-dependent mortality (that is, the small inbred plants tended to die) limiting the later age expression of ID in the DBH of surviving trees (Hardner and Potts, 1997; Waller et al., 2008). Such size-dependent mortality may result in tradeoffs between fitness surrogates, and complicate the estimation of ID for specific traits in survivors as early and late age estimates are based on a different set of trees (Williams and Savolainen, 1996; Wu et al., 1998; Armbruster and Reed, 2005). In the present case, ID in DBH of survivors was relatively stable across ages and similar on both sites. However, ID for survival accumulated with age and was markedly higher on the more productive site (Ridgley). ID has been reported to increase in more stressful environments (reviewed in Armbruster and Reed, 2005). Yet, what constitutes a more stressful environment in the present case is not clear. The less productive site at Southport could be considered more stressful for E. globulus in an abiotic sense, but with the higher mortality it could be argued that the more productive site at Ridgley is more stressful for tree growth. The reduced ID for SURV and 

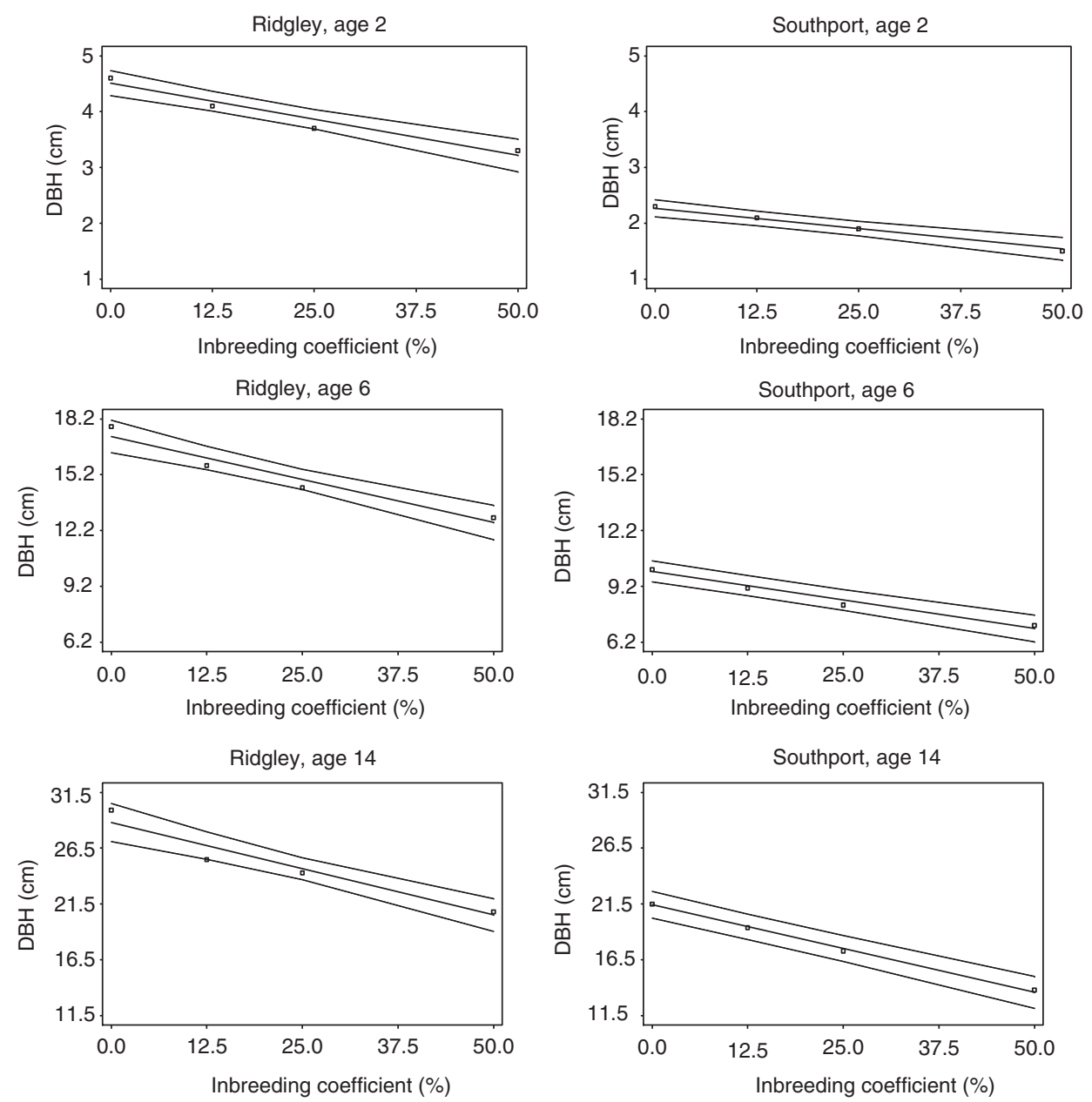

Figure 2 Fitted linear regressions (with the upper and lower bounds of 95\% confidence intervals) of DBH on the expected inbreeding coefficients $(f)$ for the Ridgley and Southport trials at ages 2, 6 and 14 years from planting. The squared symbols refer to least-squares means estimated for each of four cross types (see Table 2) corresponding to $f=0 \%$ (outcrosses), $12.5 \%$ (crosses among half sibs), 25\% (matings among full sibs) and 50\% (selfing).

reduced genetic variance for DBH under outcrossing on the least productive site are consistent with the simulations of Ronce et al. (2009), that showed reduced ID when populations are maladapted and express low overall genetic variance (see also Waller et al., 2008). However, the more likely explanation for the differences in ID for survival between sites is the greater and earlier onset of inter-tree competition for resources at the more productive and more closely planted site (Tomé et al., 1994), resulting in greater suppression of progeny exhibiting even mild inbreeding by the more vigorous outcrosses (Schmitt and Ehrhardt, 1990; Hardner and Potts, 1997; Cheptou and Mathias, 2001; Lhamo et al., 2006; Pujol and McKey, 2006). This hypothesis accords with trends observed at early stages in the E. globulus life cycle, where there is some evidence for greater selection against selfed compared with outcrossed zygotes during development when resource competition is higher (McGowen et al., 2010). Nutrient limitation has been shown to increase selection against selfs in Lupinus texensis (Fabaceae) (Helenurm and Schaal, 1996). Mixed mating systems are the norm in eucalypts (Byrne, 2008), and the present study suggests that reduced ID across the life cycle in the absence of intense intra-specific competition for resources would favour selfing as a strategy for reproductive assurance and the maintenance of mixed mating systems (Hardner and Potts, 1997; Cheptou and Mathias, 2001). Such variable ID is assumed in many models of mating system evolution (Cheptou and Mathias, 2001), but Charlesworth (2006) notes that more evidence of stronger ID under competition is required. Although the present study suggests that competition between cross types may increase the expression of ID, it is clearly not a requirement for the expression of severe ID. For example, Costa e Silva et al. (2010a) reported significant differential mortality of selfs compared with outcrosses between 4 and 6 years of age in another inbreeding experiment of E. globulus where selfs and outcrosses were not competing (see below). In this large-plot study, the reduced tree density in the selfplots with age, compared with the plots of outcrosses, resulted in a reduced ID in the DBH of survivors with age, but differential mortality continued.

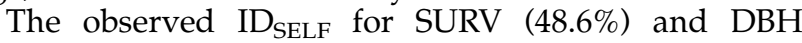
$(30.4 \%)$ at age 14 years in Ridgley is expected to be biologically important under natural conditions (Crnokrak and Roff, 1999), but is below the average ID reported for outcrossing plant species $(53 \%$, Husband and Schemske, 1996). The ID $\mathrm{IELF}_{\mathrm{S}}$ observed for the King Island population in the present study is also markedly 
Table 3 Estimated coefficients of among-family $\left(\mathrm{CV}_{\mathrm{f}}\right)$ and withinfamily $\left(\mathrm{CV}_{\mathrm{wf}}\right)$ variation for $\mathrm{DBH}$ based on progeny data from four crossing designs-outcrosses (OUT), half-sib inbreeding (HSI), full-sib inbreeding (FSI) and selfing (SELF)-in two E. globulus field trials (Ridgley and Southport) at different ages (in years) from planting

\begin{tabular}{|c|c|c|c|c|c|c|c|c|}
\hline \multirow[t]{2}{*}{ Age } & \multicolumn{4}{|c|}{$C V_{f}(\%)$} & \multicolumn{4}{|c|}{$C V_{\text {wf }}(\%)$} \\
\hline & OUT & $H S I$ & FSI & SELF & OUT & HSI & $F S I$ & SELF \\
\hline \multicolumn{9}{|c|}{ Ridgley } \\
\hline 2 & $6.5^{* * *}$ & $6.9^{* *}$ & $14.0^{* * *}$ & $18.2^{* * *}$ & 19.8 & 25.2 & 26.2 & 32.4 \\
\hline 4 & $5.6^{* * *}$ & $8.1^{* * *}$ & $11.8^{* * *}$ & $18.9^{* * *}$ & 13.6 & 18.9 & 20.4 & 24.5 \\
\hline 6 & $7.4^{* * *}$ & $8.6^{* * *}$ & $8.7^{* * * *}$ & $15.5^{* * *}$ & 15.7 & 20.3 & 20.1 & 21.8 \\
\hline 10 & $9.3^{* * *}$ & $10.0^{* * *}$ & $9.6^{* *}$ & $12.5^{* * *}$ & 19.2 & 23.2 & 25.3 & 25.4 \\
\hline 14 & $8.2^{* * *}$ & $9.9^{* * *}$ & $8.1^{\mathrm{ns}}$ & $10.1^{* *}$ & 20.1 & 25.4 & 27.3 & 27.3 \\
\hline \multicolumn{9}{|c|}{ Southport } \\
\hline 2 & $1.4^{\mathrm{ns}}$ & $14.3^{* *}$ & $9.1^{\mathrm{ns}}$ & $24.9^{* * *}$ & 42.1 & 44.4 & 48.8 & 60.4 \\
\hline 4 & $0.0^{\mathrm{a}}$ & $5.9^{\text {ns }}$ & $10.3^{*}$ & $19.6^{* * *}$ & 26.2 & 28.6 & 36.0 & 39.0 \\
\hline 6 & $0.0^{\mathrm{a}}$ & $4.3^{\mathrm{ns}}$ & $8.4^{\text {ns }}$ & $15.5^{* * *}$ & 24.0 & 28.7 & 34.9 & 33.8 \\
\hline 8 & $0.0^{\mathrm{a}}$ & $4.9^{\mathrm{ns}}$ & $2.5^{\mathrm{ns}}$ & $14.3^{* * *}$ & 23.2 & 27.9 & 31.1 & 33.6 \\
\hline 14 & $0.0^{\mathrm{a}}$ & $5.8^{\text {ns }}$ & $1.7^{\mathrm{ns}}$ & $16.0^{* * *}$ & 21.9 & 24.1 & 29.0 & 31.8 \\
\hline
\end{tabular}

a Variance estimate constrained to be non-negative at the boundary of zero.

The CV values were calculated using the estimated least-squares means (LSM) for the corresponding cross types (as presented in Table 2).

The significance probabilities given within parentheses refer to one-tailed likelihood ratio tests, used to test the departure of the estimated among-family variances from zero.

Non-significant (ns), $P>0.05$.

$* P \leqslant 0.05$.

$* * P \leqslant 0.01$.

$* * * P \leqslant 0.001$.

less than that reported for a population from a more central part of the large and continuous coastal distribution of E. globulus in south-eastern Tasmania, and tested in an adjacent field trial planted at the same time in Ridgley (Costa e Silva et al., 2010a). At 10 years of age, the ID $_{\text {SELF }}$ for SURV in the present study was 36\% (Table 1) but $74 \%$ in the adjacent trial (Costa e Silva et al., 2010a). This marked difference in magnitude of ID despite the field design employed in the present study maximizing competition among cross types, whereas the opposite was done in the adjacent trial by blocking progeny according to cross type. Although the number of founder trees from King Island is relatively low (11 base population parents), these were widely distributed, and a population bottleneck and phase of inbreeding during its recent evolutionary past (Pujol et al., 2009; Tamaki et al., 2009) is the most likely explanation for the lower ID in the King Island compared with that observed in $E$. globulus from south-eastern Tasmania. The hypothesis that the King Island population has experienced purging of deleterious recessive alleles is supported by the rangewide microsatellite DNA study of Steane et al. (2006), where the E. globulus from King Island had the lowest observed and expected heterozygosity, allelic richness and effective number of alleles.

It has been argued that rare and partially recessive deleterious alleles as opposed to overdominance drives ID in E. globulus (Costa e Silva et al., 2010a,b). The present study further argues that, at least at the population level, epistasis does not appear to be a significant contributor. ID was linearly related to the expected inbreeding coefficient for SURV (Figure 1) and
DBH (Figure 2), with no significant quadratic effects over the range of the examined $f$. For the population studied, this pattern was stable across ages and the two sites examined. This linearity of response agrees with studies involving other forest tree species (Sedgley and Griffin, 1989, pp 212-213; Matheson et al., 1995; Durel et al., 1996; Wu et al., 1998; White et al., 2007, pp 91-92). However, while a net contribution of epistasis to ID at the population level was not detected, the possibility of epistatic interactions between loci cannot be completely excluded, as epistatic effects expressed at the family level may cancel each other (Lynch and Walsh, 1998, Chapter 10). Indeed, several studies have shown significant curvilinear responses to inbreeding at the family level, but not at the overall population level (Pray and Goodnight, 1995; Dudash et al., 1997; Wu et al., 1998). Nevertheless, even with the lack of any cancelling effect, large departures from linearity are unlikely unless epistasis is very marked (Lynch and Walsh, 1998, Chapter 10).

Inbreeding has been reported to increase the variance of growth traits at multiple levels (among families, within families and/or total phenotypic) in several studies with Eucalyptus species (Griffin and Cotterill, 1988; Hardner and Potts, 1997), including E. globulus (Hardner and Potts, 1995; Costa e Silva et al., 2010a, b), as well as other tree species (Matheson et al., 1995; Durel et al., 1996; Williams and Savolainen, 1996; Wu et al., 1998). Under a purely additive model of gene action, increasing inbreeding is expected to increase amongfamily variation and reduce within-family variation (Lynch and Walsh, 1998, Chapter 10). The increase in within-family variation with increasing inbreeding found here and in other forestry experiments (see the references listed above) contradicts the additive expectation. However, under inbreeding, there may be several genetic factors that can increase among-family variation beyond that expected from an additive model, as well as (at least for early generations of inbreeding) enhance (or delay the decline of) within-family variation. Total genetic variance may increase when dominance effects that arise under inbreeding are important (Kelly, 1999; Moorad and Wade, 2005), hence augmenting variation both among and within families after inbreeding. Plant studies have indicated that significant ID was generally accompanied by an important contribution to genetic variance of dominance effects that arise with inbreeding, such as the variance due to homozygous dominance deviations (Shaw et al., 1998; Kelly and Arathi, 2003; Costa e Silva et al., 2010b). In addition, when studying the effects of inbreeding on the variation due to rare recessive alleles, Robertson (1952) observed that the additive component of the genetic variance can be enhanced within inbred lines after inbreeding, increasing to a maximum value when $f$ is close to $50 \%$ (and then declining to zero when inbreeding is complete). Epistatic variance involving additive effects may also be partially converted to additive variance after inbreeding (Goodnight, 1987, 1988), and thus will also enhance the total genetic variance. Besides these genetic factors, within-family variation may be increased following inbreeding due to an increase in the environmental component of variance if, for example, homozygous individuals are less stable in their development (Lerner, 1954) or more sensitive to the effects of competition than more heterozygous outcrosses. Nevertheless, size-dependent 
mortality will contribute to reduce the difference in variability between SELF and OUT progenies, and thus the changes in within-family variation with age may depend on the balance between the antagonistic effects of competition and mortality (Hardner and Potts, 1997).

Self-families of E. globulus have been shown to differ in ID (Costa e Silva et al., 2010b). In the present study, selffamilies differed significantly in their diameter growth at both sites and their performance was highly stable across sites (as indicated by high across-site family correlation estimates). In contrast, the variance among the outcrossed families was significant only on the more productive site. Although the environment may affect the pattern of expression of ID, the stability in selfed family performance compared with the outcrossed families suggests that the deleterious non-additive genetic effects on growth expressed under inbreeding are less sensitive to $G \times E$ interaction than the additive and non-additive effects expressed under random mating. This is particularly significant in E. globulus, as the non-additive genetic effects expressed under selfing (such as the homozygous dominance variance) may be substantially greater than both the dominance variance expressed under random mating and the additive variance (Costa e Silva et al., 2010b). The environmentally stable homozygous dominance variance is not expressed under outcrossing, but with the mixed mating system of E. globulus (Mimura et al., 2009) it is likely to be a significant driver of the performance of open-pollinated families (Costa e Silva et al., 2010b). When evaluating ID in benign and stressful environments, Armbruster and Reed (2005) suggested that it is possible that individual alleles might have general stress-resistance effects across different types of stresses, which could lead to highly correlated norms of reaction for individual inbred lines over different environments. However, the more likely explanation in the present case is the environmental insensitivity of the deleterious recessive alleles contributing to the homozygous dominance variance (for example, loss-of-function mutations associated with directional dominance; Charlesworth and Willis, 2009). As they are usually in the early stages of domestication, most $\mathrm{G} \times \mathrm{E}$ interaction studies in forest trees are based on openpollinated families (Costa e Silva et al., 2006; White et al., 2007, Chapters 8 and 12). In the case of E. globulus, there is empirical evidence that open-pollinated families derived from mixed mating in native stands are more stable in their growth performance across multiple sites than parental additive genetic effects predicted from outcross families (Hodge et al., 1996; Volker, 2002). The relative stability of deleterious homozygous dominance effects on growth suggested by the present study indicates that, for species with mixed mating systems, inbreeding effects in open-pollinated progenies may mask the significant additive $\mathrm{G} \times \mathrm{E}$ interaction in early life cycle studies. If the performance of open-pollinated families is erroneously assumed to be mainly reflecting additive genetic effects, this will lead to an underestimate of the adaptive response to environment change and the longer-term evolutionary potential of populations.

In conclusion, although ID was manifested on both the sites studied, the manner in which it was expressed varied, most likely due to differences in the timing and levels of inter-tree competition. ID for survival accumu- lated more rapidly on the more productive site; yet, at the last measurement age (14 years) survivors still exhibited significant ID, which indicates ongoing selection against even the mildest level of inbreeding. Such selection against inbred trees would suggest that, in the long term, both sites would be dominated by more vigorous outcrosses and inbreeding would be unlikely to accumulate across generations. However, for species with mixed mating systems, it is argued that environmentally stable inbreeding effects in open-pollinated progenies may tend to mask the additive $\mathrm{G} \times \mathrm{E}$ interaction for fitness traits and the adaptive response to the environment.

\section{Conflict of interest}

The authors declare no conflict of interest.

\section{Acknowledgements}

We thank Gunns Ltd (Ridgley) and Forestry Tasmania (Southport) for provision of the trial sites. Funding for crossing and trial establishment was provided by the CRC for Temperate Hardwood Forestry. Trial measurements were supported by the CRC for Sustainable Production Forestry, as well as by an Australian Research Council Linkage grant (LP0884001) partnered by the Southern Tree Breeding Association. The financial support given by Fundação para a Ciência e Tecnologia (Lisboa, Portugal) through the Ciência 2007 initiative to the senior author and by the Australian Research Council (BMP) is gratefully acknowledged.

\section{References}

Armbruster P, Reed DH (2005). Inbreeding depression in benign and stressful environments. Heredity 95: 235-242.

Bartlett MS (1947). The use of transformations. Biometrics 3: 39-52.

Botham R, Collin CL, Ashman TL (2009). Plant-mycorrhizal fungus interaction affect the expression of inbreeding depression in wild strawberry. Int J Plant Sci 170: 143-150.

Brown AR, Hosken DJ, Balloux F, Bickley LK, LePage G, Owen $\mathrm{SF}$ et al. (2009). Genetic variation, inbreeding and chemical exposure-combined effects in wildlife and critical considerations for ecotoxicology. Philos Trans R Soc Lond B Biol Sci 364: 3377-3390.

Byrne M (2008). Phylogeny, diversity and evolution of eucalypts. In: Sharma AK, Sharma A (eds). Plant Genome: Biodiversity and Evolution, vol. 1, Part E, PhanerogamsAngiosperm. Science Publishers: Enfield, pp 303-346.

Carr DE, Dudash MR (2003). Recent approaches into the genetic basis of inbreeding depression in plants. Philos Trans $R$ Soc Lond B Biol Sci 358: 1071-1084.

Charlesworth B, Charlesworth D (1999). The genetic basis of inbreeding depression. Genet Res 74: 329-340.

Charlesworth D (2006). Evolution of plant breeding systems. Curr Biol 16: R726-R735.

Charlesworth D, Charlesworth B (1987). Inbreeding depression and its evolutionary consequences. Annu Rev Ecol Syst 18: 237-268.

Charlesworth D, Willis JH (2009). The genetics of inbreeding depression. Nat Rev Genet 10: 783-796.

Cheptou PO, Mathias A (2001). Can varying inbreeding depression select for intermediary selfing rates? Am Nat 157: 361-373.

Costa e Silva J, Hardner C, Tilyard P, Pires AM, Potts BM (2010a). Effects of inbreeding on population mean performance and observational variances in Eucalyptus globulus. Ann For Sci 67 (doi:10.1051/forest/2010018). 
Costa e Silva J, Hardner C, Potts BM (2010b). Genetic variation and parental performance under inbreeding for growth in Eucalyptus globulus. Ann For Sci 67 (doi:10.1051/forest/2010019).

Costa e Silva J, Potts BM, Dutkowski GW (2006). Genotype by environment interaction for growth of Eucalyptus globulus in Australia. Tree Genet Genom 2: 61-75.

Crnokrak P, Roff DA (1999). Inbreeding depression in the wild. Heredity 83: 260-270.

Crow JF, Kimura M (1970). An Introduction to Population Genetics. Harper \& Row Publishers: New York.

Dudash MR, Carr DE, Fenster CB (1997). Five generations of enforced selfing and outcrossing in Mimulus guttatusinbreeding depression variation at the population and family level. Evolution 51: 54-65.

Durel CE, Bertin P, Kremer A (1996). Relationship between inbreeding depression and inbreeding coefficient in maritime pine (Pinus pinaster). Theor Appl Genet 92: 347-356.

Gilmour AR, Gogel BJ, Cullis BR, Thompson R (2006). ASReml User Guide Release 2.0. VSN International Ltd: Hemel Hempstead, UK.

Goodnight CJ (1987). On the effect of founder events on epistatic genetic variance. Evolution 41: 80-91.

Goodnight CJ (1988). Epistasis and the effect of founder events on the additive genetic variance. Evolution 42: 441-454.

Griffin AR, Cotterill PP (1988). Genetic variation in growth of outcrossed, selfed and open-pollinated progenies of Eucalyptus regnans and some implications for breeding strategy. Silvae Genet 37: 124-131.

Hardner CM, Potts BM (1995). Inbreeding depression and changes in variation after selfing Eucalyptus globulus subsp globulus. Silvae Genet 44: 46-54.

Hardner CM, Potts BM (1997). Post-dispersal selection under mixed-mating in Eucalyptus regnans. Evolution 51: 103-111.

Hardner CM, Potts BM, Gore PL (1998). The relationship between cross success and spatial proximity of Eucalyptus globulus ssp. globulus parents. Evolution 52: 614-618.

Helenurm K, Schaal BA (1996). Genetic load, nutrient limitation, and seed production in Lupinus texensis (Fabaceae). Am J Bot 83: 1585-1595.

Hodge GR, Volker PW, Potts BM, Owen JV (1996). A comparison of genetic information from open-pollinated and control-pollinated progeny tests in two eucalypt species. Theor Appl Genet 92: 53-63.

Husband BC, Schemske DC (1996). Evolution of the magnitude and timing of inbreeding depression in plants. Evolution 50: 54-70.

Keller LF, Waller DM (2002). Inbreeding effects in wild populations. Trends Ecol Evol 17: 230-241.

Kelly JK (1999). An experimental method for evaluating the contribution of deleterious mutations to quantitative trait variation. Genet Res 73: 263-273.

Kelly JK, Arathi HS (2003). Inbreeding and the genetic variance in floral traits of Mimulus guttatus. Heredity 90: 77-83.

Kenward MG, Roger JH (1997). Small sample inference for fixed effects from restricted maximum likelihood. Biometrics 53 983-997.

Lande R, Schemske DW (1985). The evolution of self-fertilisation and inbreeding depression in plants. I. Genetic models. Evolution 39: 24-40.

Lerner I (1954). Genetic Homeostasis. Oliver and Boyd: Edinburgh, Scotland.

Lhamo N, Ramsey M, Vaughton G (2006). Density- and frequency-dependent inbreeding depression in the Australian annual Hibiscus trionum var. vesicarius. Evol Ecol Res 8: 717-730.

Lynch M, Walsh B (1998). Genetics and Analysis of Quantitative Traits. Sinauer Associates, Inc.: Sunderland, MA, USA.
Matheson AC, White TL, Powell GR (1995). Effects of inbreeding on growth, stem form and rust resistance on Pinus elliottii. Silvae Genet 44: 37-46.

McGowen M, Vaillancourt RE, Pilbeam D, Potts BM (2010). Sources of variation in self-incompatibility in the Australian forest tree, Eucalyptus globulus. Ann Bot 105: 737-745.

Mimura M, Barbour RC, Potts BM, Vaillancourt RE, Watanabe KN (2009). Comparison of contemporary mating patterns in continuous and fragmented Eucalyptus globulus native forests. Mol Ecol 18: 4180-4192.

Moorad JA, Wade MJ (2005). A genetic interpretation of the variation in inbreeding depression. Genetics 170: 1373-1384.

Pray LA, Goodnight CJ (1995). Genetic variation in inbreeding depression in the red flour beetle Tribolium castaneum. Evolution 49: 176-188.

Pujol B, McKey D (2006). Size asymmetry in intraspecific competition and the density-dependence of inbreeding depression in a natural plant population: a case study in cassava (Manihot esculenta Crantz, Euphorbiaceae). J Evol Biol 19: 85-96.

Pujol B, Zhou SR, Vilas JS, Pannell JR (2009). Reduced inbreeding depression after species range expansion. Proc Natl Acad Sci USA 106: 15379-15383.

Ritland K (1996). Inferring the basis of inbreeding depression in plants. Genome 39: 1-8.

Robertson A (1952). The effect of inbreeding on the variation due to recessive genes. Genetics 37: 188-207.

Ronce O, Shaw FH, Rousset F, Shaw RG (2009). Is inbreeding depression lower in maladapted populations? A quantitative genetics model. Evolution 63: 1807-1819.

SAS Institute Inc. (2004). SAS/STAT 9.1 User's Guide. SAS Institute Inc.: Cary, NC.

Schmitt J, Ehrhardt DW (1990). Enhancement of inbreeding depression by dominance and suppression in Impatiens capensis. Evolution 44: 269-278.

Sedgley M, Griffin AR (1989). Sexual Reproduction of Tree Crops. Academic Press: London.

Shapiro SS, Wilk MB (1965). An analysis of variance test for normality (complete samples). Biometrika 52: 591-611.

Shaw RG, Byers DL, Shaw FH (1998). Genetic components of variation in Nemophila menziesii undergoing inbreeding: Morphology and flowering time. Genetics 150: 1649-1661.

Steane DA, Conod N, Jones RC, Vaillancourt RE, Potts BM (2006). A comparative analysis of population structure of a forest tree, Eucalyptus globulus (Myrtaceae), using microsatellite markers and quantitative traits. Tree Genet Genom 2: 30-38.

Stram DO, Lee JW (1994). Variance components testing in the longitudinal mixed effects model. Biometrics 50: 1171-1177.

Tamaki I, Ishida K, Setsuko S, Tomaru N (2009). Interpopulation variation in mating system and late-stage inbreeding depression in Magnolia stellata. Mol Ecol 18: 2365-2374.

Tomé M, Tomé JA, Araújo MC, Pereira JS (1994). Intraspecific competition in irrigated and fertilized eucalypt plantations. For Ecol Manage 69: 211-218.

Volker PW (2002). Quantitative genetics of Eucalyptus globulus, E. nitens and their $\mathrm{F}_{1}$ hybrid. PhD thesis, University of Tasmania.

Volker PW, Potts BM, Borralho NMG (2008). Genetic parameters of intra- and inter-specific hybrids of Eucalyptus globulus and E. nitens. Tree Genet Genom 4: 445-460.

Waller DM, Dole J, Bersch AJ (2008). Effects of stress and phenotypic variation on inbreeding depression in Brassica rapa. Evolution 62: 917-931.

White TL, Adams WT, Neale DB (2007). Forest Genetics. CABI: Wallingford, UK.

Williams CG, Savolainen O (1996). Inbreeding depression in conifers: implications for breeding strategy. For Sci 42: 102-117.

Wu HX, Matheson AC, Spencer D (1998). Inbreeding in Pinus radiata. I. The effect of inbreeding on growth, survival and variance. Theor Appl Genet 97: 1256-1268. 